\title{
Metastatic Meningioma: A Case Report and Literature Review
}

\author{
Caris Chong*
}

Department of Radiation Oncology, Cancer Centre, Fiona Stanley Hospital, Perth, Western Australia, Australia

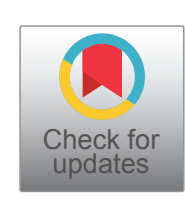

\begin{abstract}
Metastatic meningioma is extremely rare. We describe a case of a female patient who was diagnosed with metastatic meningioma on investigation of a right perihilar lung lesion. A literature search and review was performed to evaluate best management and treatment options of this condition.
\end{abstract}

Keywords

Metastatic meningioma, Literature review, Meningioma, Systemic treatment

\section{Introduction}

Although meningioma is a common and usually benign intracranial neoplasm there is a rare percentage that is atypical and more aggressive in nature. An even smaller percentage of these can be metastatic. Metastatic meningioma remains rare and published estimates range from $0.14-0.76 \%$ [1]. Extracranial spread has been reported in the literature to lung, bones, lymph nodes, liver and soft tissue $[2,3]$. There is currently no standard treatment or known optimal treatment of metastatic meningioma although there have been systemic therapies trialled in the recurrent meningioma setting which could potentially be extrapolated to the metastatic setting.

Additionally, radiation induced meningioma incidence is unknown. However, one study quoted lifetime risk as high as $22 \%$ in patients with previous cranial irradiation. Radiation induced meningiomas tend to be more aggressive and with higher rates of recurrence compared to spontaneous meningiomas [4].

\section{Methods}

We performed literature searches in Pubmed and MEDLINE for articles on metastatic meningioma published between 1971 and December 2020. Keywords and phrases used: Metastatic meningioma, octreotate, systemic treatment meningioma, atypical meningioma, anaplastic meningioma, metastasis, meningioma.

\section{Case Presentation}

A 53-year-old female presented for a CT Coronary Angiogram (CTCA) as part of general health screening as her father recently passed away from cardiac related disease. She has a background of previous childhood Non-Hodgkins
Lymphoma diagnosed in April 1977 at the age of 10-yearsold. She was treated with chemotherapy and mantle field and cranial radiotherapy and has been in remission since. Incidentally a right perihilar lung lesion was reported on CTCA (Figure 1). PET scan (F-18) was performed to further investigate this which showed photopaenic defect in the left parietal lobe of the brain (Figure 2a) and prominent FDG uptake of the right perihilar node (Figure $2 b$ ) and two other small nodules in the right upper lobe.

Patient had MRI Head performed to further characterise the lesion. A left parietal parafalcinedurally based mass measuring $35 \times 31 \times 30 \mathrm{~mm}$ was reported. Poorly defined foci were also reported in the left frontal and parietooccipital calvarium, right frontal and left petrous ridge.

Right mediastinal lesion was biopsied and pathology thought to be meningothelial meningioma. It was then resected and further histopathology showed features of a WHO Grade II Atypical Meningioma-tumour cells seen in cords and sheets, with increased nuclear cytoplasmic ratio and multiple foci of necrosis. Mitotic features 12/10 per high power field seen. Immunohistochemistry stains EMA, p63 and $C D 56$ positive. Histopathology was Pathologist peer

*Corresponding author: Dr. Caris Chong, Department of Radiation Oncology, Genesis Care, Fiona Stanley Hospital, Locked Bag 100, Palmyra DC, Western Australia 6961, Australia, Tel: (61)86152-2222; 0422-662-089, Fax: (61)89332-4471

Accepted: June 12, 2021

Published online: June 14, 2021

Citation: Chong C (2021) Metastatic Meningioma: A Case Report and Literature Review. Insights Neurooncol 4(1):48-50 


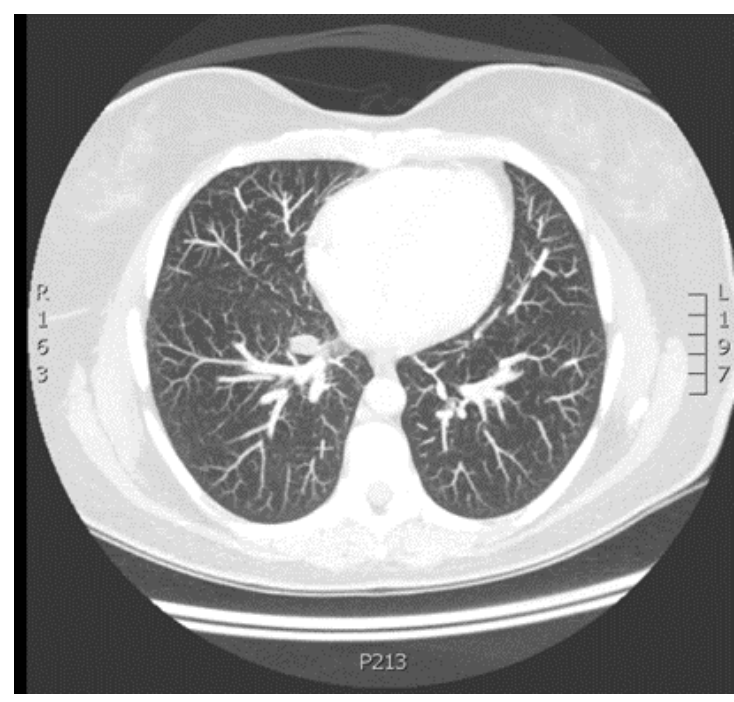

Figure 1: CT demonstrated Right perihilar lesion.

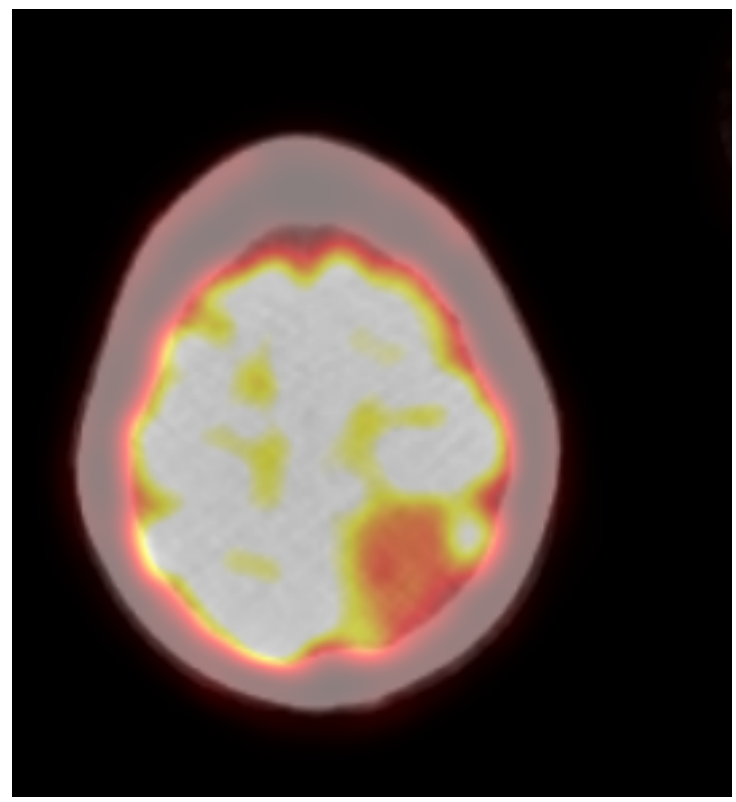

Figure 2a: PET Scan showed large photopaenic defect left parietal region.

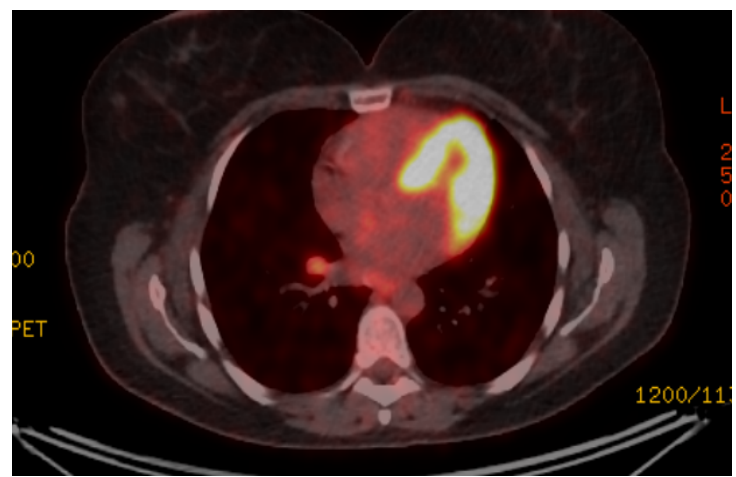

Figure 2b: PET scan showing the Right perihilar lesion with prominent FDG avidity. reviewed to ensure appropriate diagnosis given rarity of the case.

Gallium 68 Octreotate PET scan was then undertaken following the pathology results and confirmed metastatic octreotate avid meningioma. Large left parafalcine meningioma is intensely octreotate avid and thought to be the primary lesion with other metastatic foci of octreotate avid disease - two foci in right frontal bone and one in the greater wing of left sphenoid.

Debulking of the large parasagittal meningioma was performed with a small residual amount of tumour on the superior sagittal sinus seen on post-operative CT scan. Histopathology confirmed the primary tumour as a WHO Grade II Atypical Meningioma with sheet like meningothelial architecture and a lesser spindled component. This tissue was sent for Tru Sight Oncology 500 next generation molecular profiling for assessment of possible therapeutic options. Unfortunately results found no evidence of targetable nature for any potential therapeutic options. It was noted during MDT discussion that the patient showed intense somatostatin receptor uptake on her Octreotate scans and the possibility of Peptide Receptor Radiotherapy (PRRT) was discussed. She was assessed by nuclear medicine physicians and was offered PRRT with Lutetium-Ocreotate intravenously.

\section{Discussion}

Metastatic meningioma is an extremely rare entity and therefore lacking large evidence base for best management and treatment. The metastastic route is not yet established but haematogenous spread via paravertebral venous plexus appears likely especially with primary lesions that have involvement of superior sagittal sinus.

A retrospective review of 1193 patients treated for meningioma found a $0.67 \%$ rate of systemic meningioma metastasis. 28 patients were screened for metastatic disease. Out of these, 8 were diagnosed with metastatic meningioma 5 patients were WHO grade II and 3 with grade III meningioma. Of note - all patients with metastases had multiple recurrent meningiomas and undergone multimodality treatment [5]. Other studies such as Kessler, et al. have found Grade II lesions to be the primary source of metastatic disease [1], whilst Surov, et al. found Grade III lesions to be the most common cause of metastatic disease but with a spread over Grade I and II lesions as well [6]. This perhaps reflects that the WHO grading is not a good predilection as to whether the patient may have metastatic disease and that multiple recurrent disease in patients may be a greater risk factor for metastatic disease.

There are a handful of small trials in the recurrent and progression setting for meningioma involving hydroxyurea, sunitinib and bevacizumab. However, these systemic treatments have yet to be trialled in the metastatic setting. Extrapolated findings in recurrent meningioma are widely varied with limited efficacy. Potential activity of VEGF inhibitors are suggested but not yet validated. A prospective Phase II trial for refractory meningioma using temozolomide 
reported no cases of partial or complete response [7]. A RANO review looked at 47 publications with refractory meningiomas using a variety of agents including hydroxyurea, irinotecan, temozolomide, interferon-a mifepristone, octreotide analogues, megostrol acetate, imatinib, bevacizumab and gefitinib. These ranged from pilot studies to a single phase III study. The only extractable outcome was PFS 6 month rate which was $29 \%$ for WHO Grade I and $26 \%$ for WHO II/ III. It was concluded that the current medical therapies for refractory meningiomas resulted in poor outcomes [8].

There are currently two case reports in the literature of successfully treating metastatic or recurrent meningioma with liposomal doxorubucin with some response. The initial case by Read, et al. in 2003 showed near resolution of widespread pleural and lung metastasis [9] and the other by Travitzsky, et al. in 2017 showed some clinical regression of a patient with cervical spinal cord compression from recurrent tumour [10].

Recently the Pi3k/Akt/mTOR pathway was demonstrated to be hyperactivated in most meningiomas [11]. This pathway combined with the increased somastatin receptor expression were the targets of a small phase II trial CEVOREM performed in France where a combination of everolimus and octreotide in recurrent meningioma patients with no other further surgical or radiotherapy options was used. 20 patients were enrolled and published results show improved 6 month and 12 month progression free survival compared to untreated recurrent meningioma [12].

With the emergence of new targetable receptors - although still with significant evidence paucity, there are potentially more systemic options for these patients. In particular, the use of radionuclides in screening and treatment are an emerging option and has been used in a handful of patients in the literature. A recent feasibility study published in May 2020 used Somatostatin receptor directed radionuclide therapy combined with external beam radiotherapy in patients with advanced meningioma. It showed it was a feasible and safe treatment option with some good disease stabilisation [13].

\section{Conclusion}

Due to the rarity of metastatic meningioma the evidence remains limited in optimal treatment options for these patients. The use of radionuclides, as was used in this patient remains an area of potential significant efficacy and the emerging results will be an important factor in future management of recurrent and metastatic meningioma.

\section{References}

1. Kessler RA, Garzon-Muvdi T, Yang W, et al. (2017) Metastatic atypical and anaplastic meningioma: A case series and review of the literature. World Neurosurg 101: 47-56.

2. Limarzi F, Solaini L, Ercolani G, et al. (2020) Liver metastasis from a non-recurrent atypical cranial meningioma: A case report. Pathologica 112: 46-49.

3. McCarthy C, Hofer M, Vlychou M, et al. (2016) Metastatic meningioma presenting as a malignant soft tissue tumour. Clin Sarcoma Res 6: 23.

4. Banerjee J, Paakko E, Harila M, et al. (2009) Radiationinduced meningiomas: a shadow in the success story of childhood leukemia. Neuro Oncol 11: 543-549.

5. Dalle Ore CL, Magill ST, Yen AJ, et al. (2019) Meningioma metastases: Incidence and proposed screening paradigm. J Neurosurg 132: 1447-1455.

6. Surov A, Gottschling S, Bolz J, et al. (2013) Distant metastases in meningioma: An underestimated problem. J Neurooncol 112: 323-327.

7. Chamberlain MC, Tsao-Wei DD, Groshen S (2004) Temozolomide for treatment-resistant recurrent meningioma. Neurology 62: 1210-1212.

8. Kaley T, Barani I, Chamberlain M, et al. (2014) Historical benchmarks for medical therapy trials in surgery- and radiation-refractory meningioma: A RANO review. Neuro Oncol 16: 829-840.

9. Read WL, Williams F (2017) Recurrent meningioma of the cervical spine, successfully treated with liposomal doxorubicin. Case Rep Oncol 10: 656-659.

10. Travitzky M, Libson E, Nemirovsky I, et al. (2003) Doxilinduced regression of pleuro-pulmonary metastases in a patient with malignant meningioma. Anticancer Drugs 14: 247-250.

11. Johnson MD, Okedli E, Woodard A, et al. (2002) Evidence for phosphatidylinositol 3-kinase-Akt-p7S6K pathway activation and transduction of mitogenic signals by platelet-derived growth factor in meningioma cells. J Neurosurg 97: 668-675.

12. Graillon T, Sanson M, Campello C, et al. (2020) Everolimus and octreotide for patients with recurrent meningioma: results from the phase II CEVOREM trial. Clin Cancer Res 26: 552-557.

13. Hartrampf PE, Hanscheid H, Kertels O, et al. (2020) Longterm results of multimodal peptide receptor radionuclide therapy and fractionated external beam radiotherapy for treatment of advanced symptomatic meningioma. Clin Transl Radiat Oncol 22: 29-32.

DOI: $10.36959 / 828 / 336$ 